\title{
Oclusão Percutânea do Canal Arterial com Molas de Liberação Controlada
}

\author{
Roberto Max Lopes ${ }^{1}$, Francis Magalhães Gonçalves ${ }^{1}$, Maurício de Rezende Barbosa ${ }^{1}$, \\ Cristiane Nunes Martins ${ }^{1}$, Mario Osvaldo Vrandecic ${ }^{1}$
}

\section{RESUMO}

Introdução: A oclusão percutânea do canal arterial tem sido realizada com eficácia e segurança, reservando-se o tratamento cirúrgico a situações específicas tecnicamente desfavoráveis à utilização da via percutânea, como a abordagem de prematuros. O dispositivo mola de liberação controlada configurou-se como técnica segura e de baixo custo. Apresentamos a experiência de nosso serviço com esse dispositivo, discutindo aspectos técnicos do implante e características do material utilizado. Método: No período de 2002 a 2009 foram encaminhados 90 pacientes para oclusão percutânea do canal arterial. A seleção da mola a ser utilizada guardou relação com o menor diâmetro do canal arterial. Todos os pacientes foram diagnosticados em base clínica, com confirmação por meio de ecocardiografia transtorácica. Foi realizado angiograma imediatamente após o procedimento para verificação da presença de fluxo residual e de possíveis complicações imediatas. Resultados: A idade variou de 4 meses a 36 anos (mediana de 3 anos e 4 meses). O diâmetro do canal arterial variou de $0,2 \mathrm{~mm}$ a $6 \mathrm{~mm}$ (média de 2,3 mm). Os tipos de canal arterial persistente, de acordo com a classificação morfológica de Krichenko, distribuíram-se da seguinte forma: tipo A, 80 (88,8\%); tipo C, $4(4,6 \%)$; e tipo E, $6(6,6 \%)$. Obteve-se sucesso em $97,6 \%(81 / 83)$ dos casos. Não observamos nenhum caso de hemólise, endarterite ou obstruções vasculares. Observamos apenas um caso de embolização em que o dispositivo foi recapturado com o próprio cabo liberador e reposicionado com sucesso. Conclusão: $O$ presente estudo demonstrou a segurança e a eficácia do procedimento de oclusão percutânea do canal arterial.

DESCRITORES: Persistência do canal arterial. Mola de liberação controlada. Cardiopatia congênita.

1 Hospital Biocor - Nova Lima, MG, Brasil.

Correspondência: Roberto Max Lopes. Rua da Paisagem, 280 - Belo Horizonte, MG, Brasil - CEP 34000-000

E-mail: maxcordis@terra.com.br

Recebido em: 3/1/2010 • Aceito em: 1/3/2010

\section{ABSTRACT}

Percutaneous Occlusion of Patent Ductus Arteriosus with Controlled-Release Coils

Background: The percutaneous occlusion of patent ductus arteriosus is an effective and safe procedure and surgical intervention is restricted to specific unfavorable technical conditions, such as in premature infants. The controlledrelease coil has proven to be a low cost and safe method. We report our experience with this device, discussing technical aspects of the implant and coil characteristics. Method: From 2002 to 2009, 90 patients were referred for the percutaneous occlusion of patent ductus arteriosus. The size of the coil was based on the narrowest ductal luminal diameter. Diagnosis of all of the patients was based on clinical evaluation and transesophageal echocardiography. Aortic angiogram was performed immediately after the procedure to assess residual shunt and early complications. Results: Age ranged from 4 months to 36 years (median: 3 years and 4 months). The patent ductus arteriosus diameter ranged from $0.2 \mathrm{~mm}$ to $6 \mathrm{~mm}$ (mean: $2.3 \mathrm{~mm}$ ). According to Krichenko's morphological classification, the types of patent ductus arteriosus were divided as follows: type A, $80(88.8 \%)$; type C, $4(4.6 \%)$; and type E, $6(6.6 \%)$. Success was achieved in $97.6 \%(81 / 83)$ of the cases. There was no evidence of hemolysis, endarteritis or vascular obstruction. We observed only one case of embolization where the device was retrieved back into the delivery catheter and repositioned successfully. Conclusion: This study showed that the controlled-release coil is safe and effective for the percutaneous occlusion of patent ductus arteriosus.

KEY-WORDS: Ductus arteriosus, patent. Heart defects, congenital. Prostheses and implants. 
ou como método de prevenção ao desenvolvimento de endarterite bacteriana.

Desde a introdução do procedimento da oclusão percutânea do canal arterial persistente, em 1971, por Portsmann et al., ${ }^{2}$ vários dispositivos e técnicas de oclusão vêm sendo desenvolvidos. Nesse período, o fechamento percutâneo do canal arterial persistente tornou-se método terapêutico consolidado, reservando-se o tratamento cirúrgico à abordagem de prematuros de muito baixo peso, lactentes sintomáticos com peso inferior a $5 \mathrm{~kg}$ e pacientes portadores de amplos canais arteriais associados a outras cardiopatias não passíveis de tratamento percutâneo.

O dispositivo mola de liberação controlada tem sua eficácia e segurança bem estabelecidas para a oclusão percutânea de canais arteriais pequenos e moderados ( $<4 \mathrm{~mm}$ ). Os dispositivos do tipo Nit Occlud e o oclusor ductal de Amplatzer são utilizados com maior eficácia em canais de diâmetros maiores ou de morfologia desfavorável à oclusão com molas. ${ }^{3}$

Apresentaremos nossa experiência com o dispositivo mola de liberação controlada Flipper (William Cook, Bjaeverskov, Dinamarca).

\section{MÉTODO}

Trata-se de estudo observacional prospectivo em que foram revisados os prontuários dos pacientes portadores de canal arterial persistente, selecionados para oclusão percutânea, entre janeiro de 2002 e dezembro de 2009. Nesse período, 90 pacientes consecutivos foram encaminhados para tentativa de oclusão percutânea do canal arterial com molas de liberação controlada.

Os critérios de exclusão utilizados foram: prematuridade, pacientes com peso abaixo de $5 \mathrm{~kg}$, presença de cardiopatia congênita associada não passível de abordagem percutânea, canais muito curtos ou do tipo janela, doenças infecciosas ativas e presença de hipertensão pulmonar fixa.

Foram incluídos pacientes com diagnóstico de canal arterial persistente por meio da ecocardiografia transtorácica com repercussão hemodinâmica. Pacientes portadores de canais arteriais pequenos, sem repercussão hemodinâmica, também foram encaminhados para terapia percutânea como método profilático para o desenvolvimento de endarterite bacteriana. Nesses casos o diagnóstico do canal arterial se deu durante a propedêutica de sopros cardíacos em pacientes assintomáticos.

Os pacientes foram submetidos a anestesia geral ou sedação consciente. Todos receberam profilaxia antibiótica com uso de cefazolina. O acesso foi obtido por meio de punção de veia e artéria femorais seguida de heparinização sistêmica.
Após a aquisição de dados hemodinâmicos realizamos aortografias nas projeções lateral esquerda e/ou oblíqua anterior direita. A partir das angiografias procedemos com cuidadosa caracterização morfológica do canal arterial de acordo com a classificação de Krichenco, utilizando-se o dispositivo Flipper. O diâmetro interno variou de $3 \mathrm{~mm}$ a $8 \mathrm{~mm}$ e o número de alças, de 3 a 5 . A seleção das molas baseou-se no diâmetro mínimo do canal arterial. O diâmetro helicoidal da mola selecionada manteve relação de duas a duas vezes e meia o diâmetro mínimo do canal arterial. Preferencialmente as molas foram liberadas por via anterógrada, reservando-se a via retrógrada para a abordagem de canais com diâmetros com menos de $1 \mathrm{~mm}$ ou em caso de necessidade de implante consecutivo de uma segunda mola para tratamento de fluxos residuais. Nos casos em que antecipamos a necessidade de implante de duas molas (canais $>3,5 \mathrm{~mm}$ ), optamos pela estratégia de implante por via anterógrada com a utilização de acesso venoso femoral bilateral (Figura 1). Após o posicionamento anterógrado de dois cateteres multipurpose $5 \mathrm{~F}$ na aorta descendente, introduzimos o cabo de liberação, exteriorizando as molas em configuração helicoidal através da retração do mandril. Posteriormente, as molas foram tracionadas simultaneamente para dentro da ampola do canal arterial, exteriorizando uma alça na artéria pulmonar com manutenção das demais no interior do canal arterial.

Dessa maneira, adquirimos melhor compactação do material trombogênico no interior do canal, associada a maior estabilidade da prótese. Nesses casos, a segunda mola guardou relação de uma vez e meia a duas vezes o diâmetro mínimo do canal. Um segundo angiograma foi realizado imediatamente após o procedimento para avaliar a presença ou não de fluxo residual. O fluxo residual foi caracterizado como fluxo difuso de baixa velocidade (enevoado) ou fluxo localizado de alta velocidade em jato. ${ }^{4}$ Após a realização do aortograma de controle, retiraram-se os cateteres e realizou-se hemostasia por compressão manual.

Considerou-se sucesso do procedimento quando ocorreu o correto posicionamento da mola no interior do canal arterial, associado à oclusão angiográfica total do canal arterial ou à presença de fluxo residual difuso de baixa velocidade. Pequenos fluxos residuais sem formação de jatos foram conduzidos de maneira conservadora sem implante de uma segunda mola. Todos os pacientes foram submetidos a estudo ecocardiográfico cerca de 24 horas após o procedimento.

O acompanhamento ecocardiográfico foi importante para detecção de fluxos residuais, monitorização da reabertura de canais, bem como para ocorrência de estreitamentos vasculares na aorta torácica e no ramo esquerdo da artéria pulmonar. Neste caso, considerouse gradiente vascular significativo a constatação de velocidades de fluxo maiores que $2 \mathrm{~m} / \mathrm{s}$ através dos vasos. 


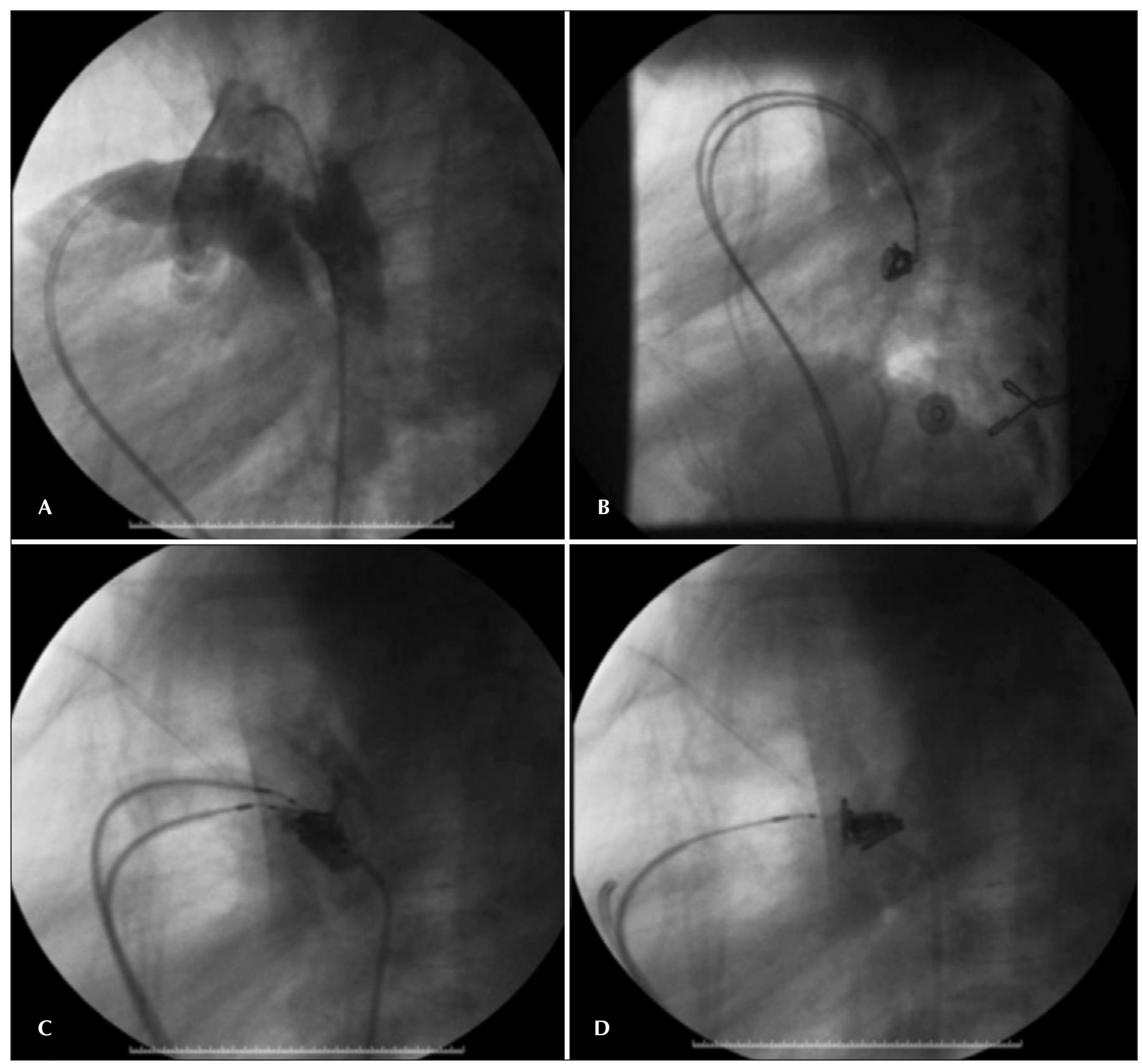

Figura 1 - Implante simultâneo por via anterógrada. Em A, aortografia em perfil esquerdo. Em B, molas espiraladas simultaneamente na aorta descendente. Em C, molas posicionadas no interior da ampola do canal arterial. Em D, molas liberadas.

\section{RESULTADOS}

Foram selecionados 90 pacientes, sendo 60 do sexo feminino. A faixa etária variou de 4 meses de vida a 36 anos de idade (mediana de 3 anos e 4 meses). Apenas 4 pacientes apresentavam idade superior a 18 anos. O peso variou entre $5 \mathrm{~kg}$ e $60 \mathrm{~kg}$ (mediana de 10,2 kg).

Considerando-se o total de 90 pacientes encaminhados para oclusão percutânea do canal arterial persistente, o diâmetro do canal arterial em sua extremidade pulmonar variou de $0,2 \mathrm{~mm}$ a $6 \mathrm{~mm}$ (média de $2,3 \mathrm{~mm}$; desvio padrão $\pm 0,37$ ) (Figura 2); 62 pacientes $(69 \%)$ apresentaram canal arterial persistente $\leq 2,5 \mathrm{~mm}$; 28 pacientes (31\%) apresentaram canal arterial persistente $>2,5 \mathrm{~mm}$; e 9 pacientes apresentaram canal arterial $\geq 4 \mathrm{~mm}, 7$ desses canais medindo $4 \mathrm{~mm}$ e 2 medindo $5 \mathrm{~mm}$.

Os tipos de canal arterial persistente, de acordo com a classificação morfológica de Krichenko et al., ${ }^{5}$ distribuíram-se da seguinte forma: tipo A, 80 (88,8\%); tipo C, 4 (4,6\%); e tipo E, $6(6,6 \%)$.

A pressão média na artéria pulmonar variou de $8 \mathrm{mmHg}$ a $43 \mathrm{mmHg}$ (mediana de 12,7 mmHg).

Dos 90 pacientes, 81 foram submetidos a oclusão percutânea do canal arterial persistente com sucesso. 
Sete pacientes foram encaminhados para reparo cirúrgico por terem apresentado canais amplos e de morfologia inadequada; um paciente foi encaminhado para cirurgia após tentativa de implante sem sucesso (risco de embolização); e outro caso, considerado insucesso por presença de fluxo residual localizado de alta velocidade em jato, apresentou canal arterial persistente de $4 \mathrm{~mm}$, permanecendo em acompanhamento ecocardiográfico ambulatorial. O procedimento foi realizado com sucesso em 97,6\% (81/83) dos casos. A angiografia realizada imediatamente após o procedimento demonstrou: ausência de fluxo residual em 43,5\% (36/83), fluxo residual difuso de baixa velocidade (enevoado) em 55,5\% (46/83), e fluxo residual localizado de alta velocidade de jato em 1\% (1/83). Todos os 47 pacientes que apresentaram fluxo residual foram submetidos a ecocardiografia após 24 horas do procedimento, dos quais 36 pacientes $(76,5 \%)$ demonstraram ausência de fluxo residual e $11(23,5 \%)$, fluxo residual de baixa velocidade. Portanto 86,7\% (72/83) dos pacientes submetidos a oclusão percutânea do canal arterial persistente apresentaram fechamento completo do canal arterial após avaliação em 24 horas (Figura 3).

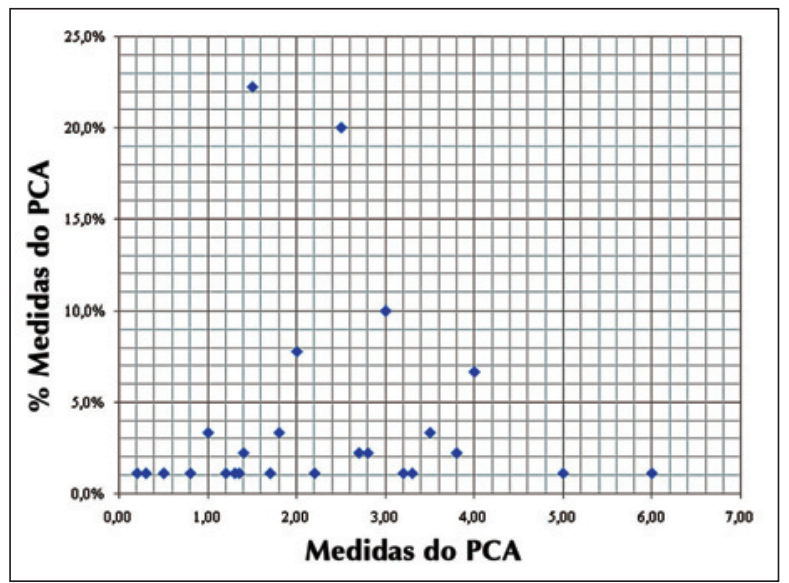

Figura 2 - Distribuição porcentual das medidas do canal arterial persistente (PCA).

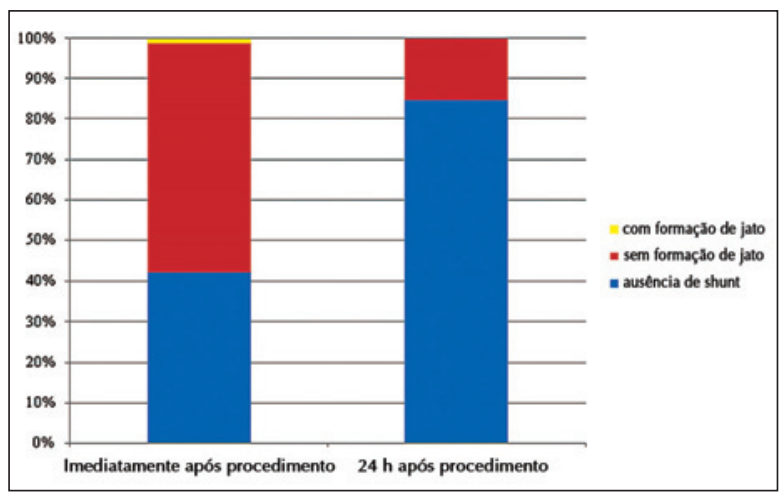

Figura 3 - Avaliação do shunt após o procedimento.
Quatro pacientes apresentaram lesão cardíaca associada, sendo três portadores de comunicações interventriculares pequenas e um com estenose pulmonar (tratada simultaneamente).

Dos 8 pacientes encaminhados para ligadura (cirúrgica) do canal arterial, 7 apresentaram canais arteriais persistentes $\geq 4 \mathrm{~mm}$ e apenas um apresentou canal arterial persistente de $3,5 \mathrm{~mm}$, associado a hipertensão pulmonar (pressão média na artéria pulmonar de $33 \mathrm{mmHg}$ ). A hipertensão pulmonar estava presente em 4 pacientes cirúrgicos.

Apenas um paciente apresentou embolização do dispositivo para a artéria femoral esquerda, que foi resgatada e reposicionada adequadamente no canal arterial (Figura 4).

Implantaram-se duas molas em 10 casos, com o diâmetro mínimo do canal variando entre $2,7 \mathrm{~mm}$ e $4 \mathrm{~mm}$. Destes, 9 apresentaram morfologia do tipo A e 1, do tipo E.

O ecocardiograma mostrou aumento da velocidade de fluxo na porção ístmica (aórtica) $>2 \mathrm{~m} / \mathrm{s}$, ou seja, com potencial risco de obstrução vascular, em um caso. Esse paciente tinha menos de um ano de idade e atualmente encontra-se em acompanhamento ambulatorial.

\section{DISCUSSÃO}

A oclusão completa do canal arterial persistente imediatamente após o procedimento com a utilização de molas varia de $33 \%$ a $90 \%$, de acordo com a experiência de cada serviço. ${ }^{6}$

Kumar et al. ${ }^{7}$ apresentaram eficácia de $41 \%$ de oclusão completa e 50\% com fluxo residual difuso de baixa velocidade imediatamente após o procedimento. Na mesma série, o acompanhamento ecocardiográfico em 24 horas evidenciou fechamento completo em $92 \%$ dos casos e após 3 meses, em 100\%. Arora et al. ${ }^{8}$ relataram $20 \%$ de fluxo residual após 24 horas, e Uzun et al. ${ }^{9}$ citam $60 \%$ de fechamento completo imediatamente após o procedimento.

A reabertura do canal arterial após o sucesso inicial com molas de Gianturco foi relatada principalmente quando esse dispositivo foi utilizado em canais curtos ou de morfologia tipo B segundo a classificação de Krichenco. ${ }^{10}$ Acreditamos que esse dispositivo não seja adequado para o tratamento desses tipos morfológicos. Não observamos esse evento no seguimento de nossa casuística, provavelmente em decorrência de nossos critérios morfológicos de exclusão. No caso de utilização de molas para a abordagem desses tipos morfológicos, é importante que esses pacientes sejam mantidos em rigoroso seguimento ambulatorial.

A oclusão percutânea do canal arterial persistente oferece vantagens em relação à cirurgia de ligadura/ 

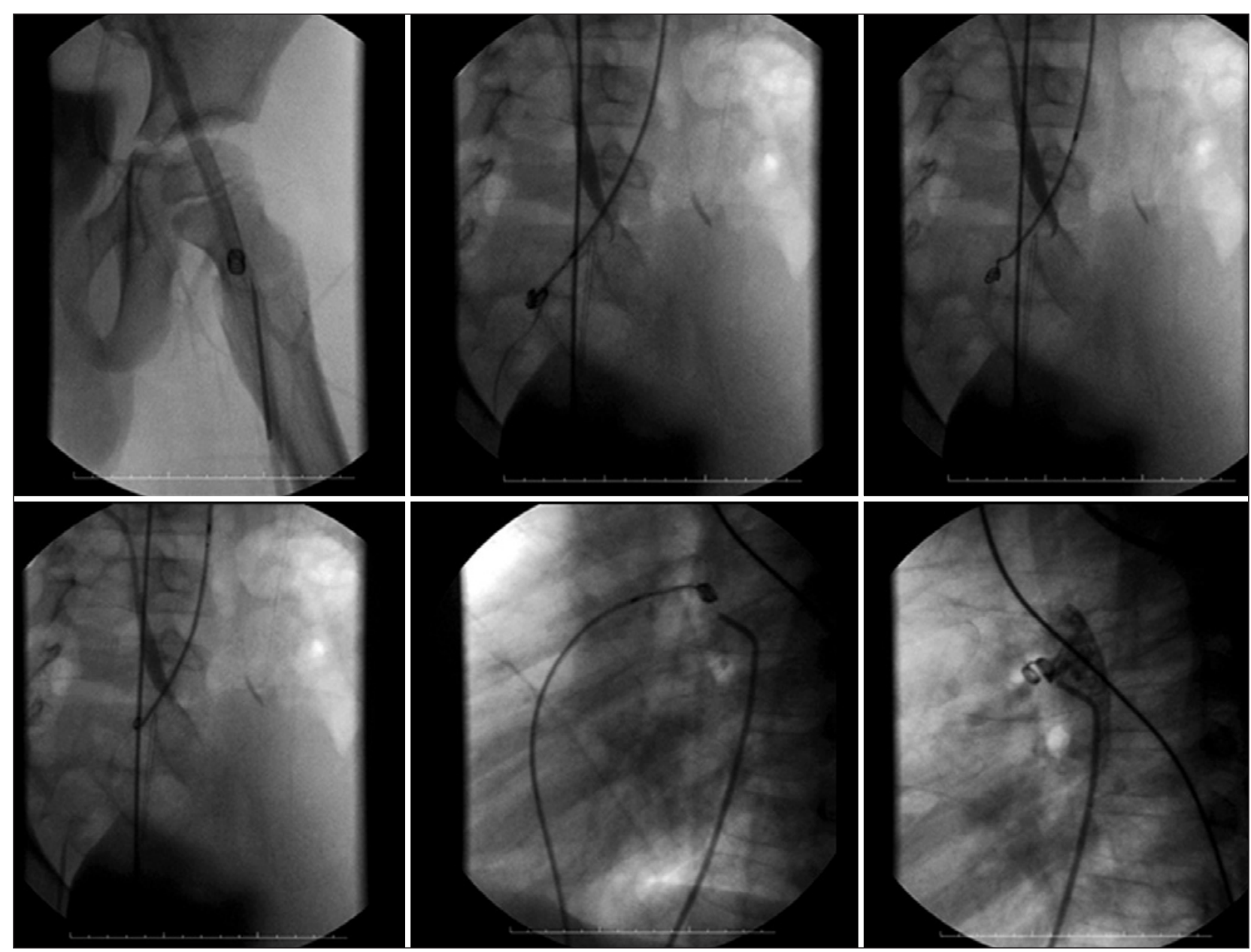

Figura 4 - Painel superior: à esquerda, em PA, imagem da mola embolizada na artéria femoral esquerda; no centro e à direita, em oblíqua anterior direita, a mola encontra-se na artéria ilíaca comum, sendo resgatada através do sistema liberador. Painel inferior: à esquerda, em oblíqua anterior direita, a mola recolhida ao cabo liberador; no centro, em perfil esquerdo, o dispositivo sendo posicionado no canal arterial; e à direita, em perfil esquerdo, a mola sendo posicionada adequadamente e ausência de shunt residual.

secção do canal, tais como: maior conforto, menor dor, menor tempo de internação e ausência de cicatriz.

O dispositivo do tipo mola de liberação controlada permite abordagem mais segura no que diz respeito ao correto posicionamento da prótese dentro do canal arterial e, consequentemente, com menor índice de embolização, como comprovado em nossa casuística. Além disso, esse dispositivo não necessita utilização de bainhas longas, que aumentam o risco de complicações vasculares. ${ }^{11} \mathrm{Na}$ abordagem de canais $>3,5 \mathrm{~mm}$, as molas de Gianturco de 0,052 polegada, liberadas com auxílio de biótomos, constituem boa alternativa, minimizando a necessidade de implante de múltiplas molas, pois apresentam maior força radial quando comparadas às molas de 0,032 polegada e de 0,038 polegada, que constituem as molas de liberação controlada. ${ }^{12}$

Dos 28 pacientes com canal arterial persistente $>2,5 \mathrm{~mm}, 21$ foram submetidos a oclusão percutânea do canal arterial com sucesso. Isso demonstra a segu- rança da abordagem de canais maiores com esse tipo de dispositivo, tornando o método acessível a maior número de pacientes, pois trata-se de dispositivo coberto pelo sistema público de saúde do País.

Não observamos, em nossa casuística, a ocorrência de hemólise. Os dados de literatura apontam incidência de cerca de $3 \%$ a $7 \% .^{13}$ Tal achado se correlaciona com a presença de fluxo residual de alta velocidade. Seu tratamento inclui infusão de haptoglobina para prevenção de insuficiência renal. ${ }^{14}$

Registramos a ocorrência de um único caso de embolização não relacionada à escolha inadequada do dispositivo (a mola não foi corretamente acoplada ao cabo liberador). A mola foi resgatada por via anterógrada em um ramo da artéria femoral esquerda com auxílio do próprio cabo liberador, sendo posteriormente posicionada no canal arterial com sucesso.

Os estreitamentos vasculares do istmo da aorta e do ramo esquerdo da artéria pulmonar são complica- 
ções potenciais relacionadas ao uso de dispositivos oclusores. ${ }^{15}$ Isso ocorre principalmente na abordagem de lactentes portadores de canais amplos, canais curtos e quando se faz necessária a utilização de múltiplas molas. A incidência de estenose do ramo esquerdo da artéria pulmonar pode estar superestimada nas séries de casos publicadas, uma vez que há dificuldade na demonstração ecocardiográfica da estenose de ramo esquerdo da artéria pulmonar na presença do fluxo através do canal (desmascarado após sua oclusão). Os gradientes aferidos no istmo da aorta se relacionam à protrusão do dispositivo. Raramente levam à necessidade de explante da prótese, desaparecendo com o crescimento somático da criança. Esse tipo de complicação é mais comum em dispositivos que utilizam disco de retenção ou quando se selecionam dispositivos de molas com diâmetro helicoidal maior que diâmetro da ampola aórtica. Outra complicação descrita na literatura médica é a lesão do nervo laríngeo recorrente, observada e relatada principalmente na abordagem de canais longos do tipo E de Krichenco. ${ }^{16}$ A fisiopatologia está relacionada ao encurtamento e a distorções anatômicas induzidas pelos dispositivos. Essa complicação não foi verificada em nossa casuística.

A maioria dos pacientes desta casuística encontra-se em acompanhamento ambulatorial. Não observamos, nesse período, a reabertura do canal arterial. Foi verificada a permanência de fluxos residuais em seguimento tardio (> 10 meses) em 2 pacientes. Em um deles o fluxo foi considerado significativo, tendose optado pelo implante de nova mola.

\section{CONCLUSÃO}

A oclusão percutânea do canal arterial com o dispositivo mola de liberação controlada Flipper (Cook), em nossa casuística, demonstrou-se segura e eficaz, sendo critério relevante a ausência de complicações do procedimento.

Não há consenso sobre quando tratar fluxos residuais difusos em relação a complicações futuras relacionados ao canal arterial persistente.

De qualquer forma, o seguimento de tais pacientes é de suma importância no que diz respeito, principalmente, à reabertura de canais após o procedimento de oclusão percutânea prévia.

\section{CONFLITO DE INTERESSES}

Os autores declararam inexistência de conflito de interesses relacionado a este manuscrito.

\section{REFERÊNCIAS}

1. Azhar AS, El-Azim AABD, Habib HS. Transcatheter closure of patent ductus arteriosus: evaluating the effect of the learning curve on the outcome. Ann Pediatr Cardiol. 2009; 2:36-40.

2. Portsmann W, Wierny L, Warnke H, Gertberger G, Romaniuk PA. Catheter closure of patent ductus arteriosus, 62 cases without thoracotomy. Radiol Clin North Am. 1971;9:201-13.

3. Haddad J, Lima Filho MO, Figueiredo GL, Nazzetta HE, Osterne ECV. Oclusão percutânea da persistência do canal arterial. Rev Bras Cardiol Invasiva. 2005;13:206-18.

4. Pedra CAC, Pedra SRFF, Esteves CA, Braga SL, Silva MA, de Almeida TL, et al. Experiência global no fechamento percutâneo do canal arterial. Arq Bras Cardiol. 1998;71(6):769-80.

5. Krichenko A, Benson LN, Burrows P, Moes CA, McLaughlin $P$, Freedom RM. Angiographic classification of the isolated, persistently patent ductus arteriosus and implications for percutaneous catheter occlusion. Am J Cardiol. 1989;63: $877-9$.

6. Lloyd TR, Fedderly R, Mendelsohn AM, Sandhu SK, Beckman $\mathrm{RH}$ III. Transcatheter occlusion of patent ductus arteriosus with Gianturco coils. Circulation. 1993;88:1412-20.

7. Kumar AS, Kapor A, Sinha N, Shahi M, Goel PK, Radhakrishnan $\mathrm{S}$, et al. Percutaneous occlusion of patent ductus arteriosus with controlled-release coil. Asian Cardiovasc Thorac Ann. 1999;7:204-8.

8. Arora R, Verma PK, Trehan V, Pandey R, Nigam H, Kalra GS. Transcatheter coil occlusion of PDA by detachable steel coils: short-term results. Indian Heart J. 1997;49:60-4.

9. Uzun O, Hancock S, Parson JM, Dikcinson DF, Gibbs JL. Transcatheter occlusion of the arterial duct with Cook detachable coils: early experience. Heart. 1996;76:269-73.

10. Daniels CJ, Cassidy SC, Teske DW, Wheller JJ, Allen HD. Reopening after successful coil occlusion for patent ductus arteriosus. J Am Coll Cardiol. 1998;31:444-50.

11. Chamié F, Chamié D, Ramos S. Oclusão percutânea dos canais arteriais com prótese Amplatzer. Rev Bras Cardiol Invasiva. 2007;15:15-24.

12. Tomita H, Ono Y, Miyazaki A, Tanaka T, Kimura K, Echigo $\mathrm{S}$. Transcatheter occlusion of patent ductus arteriosus using a 0.052-inch coil: immediate results. Jpn Circ J. 2000;64: 520-3.

13. Anil SR, Sivakumar K, Philip AK, Francis E, Kumar RK. Clinical course and management strategies for hemolysis after transcatheter closure of patent arterial ducts. Cathet Cardiovasc Interv. 2003;59:538-43.

14. Eda K, Ohtsuka S, Seo Y, Yamada S, Ishiyama M, Miyamoto $\mathrm{H}$, et al. Conservative treatment of hemolytic complication following coil embolization in two adult cases of patent ductus arteriosus. Jpn Circ J. 2001;65:834-6.

15. Jang GY, Son CS, Lee JW, Lee JY, Kim SJ. Complications after transcatheter closure of patent ductus arteriosus. J Korean Med Sci. 2007;22:484-90.

16. Liang CD, Ko SF, Huang SC, Huang CF, Niu CK. Vocal cord paralysis after transcatheter coil embolization of patent ductus arteriosus. Am Heart J. 2003;146:367-71. 